\title{
A simple clinical assessment is superior to systematic triage in prediction of mortality in the emergency department
}

\author{
Anne Kristine Servais Iversen, ${ }^{1}$ Michael Kristensen, ${ }^{2}$ Rebecca Monett Østervig, ${ }^{3}$ \\ Lars Køber, ${ }_{1}^{4}$ György Sölétormos, ${ }_{1}^{5}$ Jakob Lundager Forberg, ${ }^{6}$ Jesper Eugen-Olsen, ${ }^{7}$ \\ Lars Simon Rasmussen, ${ }^{3}$ Morten Schou, ${ }^{8}$ Kasper Karmark Iversen ${ }^{8}$
}

\begin{abstract}
- Additional material is published online only. To view please visit the journal online (http://dx.doi.org/10.1136/ emermed-2016-206382).

For numbered affiliations see end of article.

\section{Correspondence to}

Dr Anne Kristine Servais Iversen, Department of Obstetrics and Gynecology, Rigshospitalet, Kobenhavn 2200, Denmark; anneksiversen@gmail.com
\end{abstract}

The abstract was presented as a poster at the Danish Emergency Medicine Conference, Odense, Denmark, 20-21 November 2014, as well as the American Academy of Emergency Medicine, Scientific Assembly, Austin, Texas, USA, 28 February to 4 March 2015

Received 2 December 2016 Revised 9 May 2018 Accepted 18 May 2018 Published Online First 16 October 2018

\section{Linked}

- http://dx.doi.org/10.1136/ emermed-2018-207659

\section{Check for updates}

To cite: Iversen AKS Kristensen M, Østervig RM, et al. Emerg Med $\mathrm{J}$ 2019:36:66-71.

\begin{abstract}
Objective To compare the Danish Emergency Process Triage (DEPT) with a quick clinical assessment (Eyeball triage) as predictors of short-term mortality in patients in the emergency department (ED).
\end{abstract}

Methods The investigation was designed as a prospective cohort study conducted at North Zealand University Hospital. All patient visits to the ED from September 2013 to December 2013 except minor injuries were included. DEPT was performed by nurses. Eyeball triage was a quick non-systematic clinical assessment based on patient appearance performed by phlebotomists. Both triage methods categorised patients as green (not urgent), yellow, orange or red (most urgent). Primary analysis assessed the association between triage level and 30-day mortality for each triage method. Secondary analyses investigated the relation between triage level and 48-hour mortality as well as the agreement between DEPT and Eyeball triage.

Results A total of 6383 patient visits were included. DEPT was performed for 6290 (98.5\%) and Eyeball triage for $6382(\sim 100 \%)$ of the patient visits. Only patients with both triage assessments were included. The hazard ratio (HR) for 48-hour mortality for patients categorised as yellow was $0.9(95 \% \mathrm{Cl} 0.4$ to 1.9$)$ for DEPT compared with 4.2 ( $95 \% \mathrm{Cl} 1.2$ to 14.6$)$ for Eyeball triage (green is reference). For orange the HR for DEPT was $2.2(95 \% \mathrm{Cl} 1.1$ to 4.4$)$ and $17.1(95 \% \mathrm{Cl}$ 5.1 to 57.1) for Eyeball triage. For red the HR was 30.9 (95\% Cl 12.3 to 77.4$)$ for DEPT and $128.7(95 \% \mathrm{Cl} 37.9$ to 436.8) for Eyeball triage. For 30-day mortality the HR for patients categorised as yellow was $1.7(95 \% \mathrm{Cl} 1.2$ to 2.4) for DEPT and 2.4 (95\% Cl 1.6 to 3.5) for Eyeball triage. For orange the HR was $2.6(95 \% \mathrm{Cl} 1.8$ to 3.6$)$ for DEPT and 7.6 ( $95 \% \mathrm{CI} 5.1$ to 11.2$)$ for Eyeball triage, and for red the HR was $19.1(95 \% \mathrm{Cl} 10.4$ to 35.2$)$ for DEPT and 27.1 ( $95 \% \mathrm{Cl} 16.9$ to 43.5 ) for Eyeball triage. Agreement between the two systems was poor (kappa 0.05).

Conclusion Agreement between formalised triage and clinical assessment is poor. A simple clinical assessment by phlebotomists is superior to a formalised triage system to predict short-term mortality in ED patients.

\section{INTRODUCTION}

The intention behind triage is to prioritise patients in terms of their clinical urgency and to divide them into categories of acuity. ${ }^{1}$ Prior to introduction of formalised triage in emergency

\section{Key messages}

What is already known on this subject

- Studies investigating the validity of triage scales have primarily shown that triage level to some degree correlates with outcome measures such as resource use, length of stay, admission, hospital charges and mortality.

- Very few data exist on how a clinical assessment compares with formally structured triage with regard to predicting acuity.

\section{What this study adds}

- Our study suggests that a basic clinical assessment is superior to a formalised triage algorithm in predicting mortality in an unselected group of patients presenting to an emergency department.

departments (EDs), patients were prioritised based on clinical assumption in the form of informally structured triage. ${ }^{2}$ Since the 1970 s, ${ }^{3}$ several formalised triage systems have evolved and different scales are in use all over the world. ${ }^{3-5}$

The majority of triage systems are derived from expert opinion and supported by limited scientific research. ${ }^{45}$ Several studies investigating triage scales have methodological limitations and previous research has concluded that most triage scales are supported by 'limited and often insufficient evidence'. ${ }^{4}$ To date there is no universal consensus on which parameters to include in a formalised triage system. ${ }^{45}$ Because there is no agreed proxy for 'acuity', the validation of formalised triage has focused on investigating predictive validity-that is, association between triage category and different outcome measures such as resource use, length of stay, admission, hospital charges and mortality. ${ }^{5-8}$ Currently there is no evidence establishing that formalised triage is superior to informally structured triage with regard to the prediction of clinical endpoints. ${ }^{7}$ The purpose of this study was to investigate how a simple clinical evaluation (ie, Eyeball triage) compares with a presently used formalised triage system and to determine how accurate each method is in predicting short-term mortality in ED patients. 


\section{METHOD}

\section{Study design and participants}

All data derive from the 'Triage study', which is a prospective observational cohort study previously described in detail elsewhere. ${ }^{9}$ The aim of the study was to optimise risk stratification and develop methods to identify low-risk patients appropriate for early ED discharge.

The 'Triage study' was performed at North Zealand University Hospital, which is a regional hospital in the Capital Region of Denmark. The hospital has a 24 hours acute care facility offering emergency level 2 trauma, medical, surgical and intensive care services for 310000 citizens in North Zealand. The ED has approximately 68000 patient contacts annually and handles all patients except level 1 trauma patients, patients with identified ST-segment elevation myocardial infarction in the prehospital setting and hospital preferences by the patients. ${ }^{10}$ The 'Triage study' included all patients visiting the ED during the period from September 2013 to December 2013. Inclusion criteria were age $>16$ years. Patients with minor complaints and injuries were excluded (ie, no patients in the least acute triage group (blue) were included). For 108 (1.7\%) patient visits there was a loss to follow-up mainly due to emigration and patients seen in the ED without a Danish Central Person Registry number.

\section{Triage standard}

North Zealand University Hospital introduced the use of formalised triage in 2009 and since 2011 Danish Emergency Process Triage $(\mathrm{DEPT})^{3}$ has been the triage standard. Trained nurses perform triage before beginning diagnostics and before the patient is seen by a physician. DEPT ranks patients into five colour-coded triage categories. Each patient is assigned a triage level based on the two main descriptors: (1) vital signs and (2) presenting complaint. The more urgent of these two determines the final triage category. The nurse can categorise the patient to a triage category of higher acuity, one level above that determined by DEPT, if she believes the patient would benefit from a higher priority. Triage to a lower triage category than prescribed by DEPT can only occur after the patient has been seen by a physician. ${ }^{11}$ The triage categories are: (1) red (resuscitation, constant re-evaluation); (2) orange (emergent, re-evaluation every $15 \mathrm{~min}$ ); (3) yellow (potentially unstable, re-evaluation every $60 \mathrm{~min}$ ); (4) green (non-urgent, re-evaluation every $180 \mathrm{~min}$ ); and blue (minor injuries or complaints, re-evaluation every $240 \mathrm{~min}$ ). The resulting triage category is used to initiate some immediate tests and other diagnostic procedures. DEPT is currently the most widely used triage system in Denmark ${ }^{7}$ and has previously been described in detail. ${ }^{11}$ DEPT is inspired by and almost identical to the Swedish Adaptive Process Triage model (ADAPT) ${ }^{12} 13$ which has an approach to patient sorting identical to the internationally established Canadian Triage and Acuity Scale (CTAS). ${ }^{3}$

Sixty-four nurses performed DEPT during the data inclusion period. The nurses had different levels of experience but all had attended formal training in the use of DEPT before working in the ED.

\section{Eyeball triage}

Eyeball triage was performed as an immediate clinical assessment by hospital staff without formal training in patient evaluation or experience with formalised triage. The staff performing Eyeball triage consisted of a group of 10 trained phlebotomists and 10 medical students working as phlebotomists from the Department of Clinical Biochemistry. Raters were instructed to base their Eyeball triage on a quick look at the patient and if possible to ask the patient for their main complaint. Phlebotomists were instructed to assess the patients based on acuity with regard to who they believed required the most immediate attention and treatment. Eyeball triage applied the same categorisation as DEPT and raters were asked to allocate the patients into the same colour-coded categories. None of the persons performing Eyeball triage were informed of the endpoint (ie, mortality) chosen in the study. The Eyeball triage was performed in immediate succession to DEPT and blinded from the resulting DEPT category including any information obtained during triage.

\section{Data collection}

A TRIAGE database was built using the following entries:

\section{DEPT data}

During triage the nurse registered the DEPT category using the programme CETREA Emergency, Version 3.11.

\section{Eyeball triage}

Eyeball triage category was manually filled out on a paper form during patient assessment and later entered into the TRIAGE database. Random quality checks were carried out to ensure that entries were registered correctly.

\section{Mortality}

Information about vital status was obtained from the Danish Central Office of Civil Registration which continuously records the vital status of all Danish residents.

Patients were identified and all data merged using the Central Personal Registry (CPR) number which uniquely identifies all Danish citizens.

\section{Statistical analysis}

Patients who were not assessed by both triage methods were excluded from the statistical analysis.

The baseline characteristics of the population were reported using descriptive statistics.

The primary analysis assessed the association between triage category and 30-day mortality. Secondary analyses assessed the association between triage category and 48-hour mortality and also investigated the agreement between DEPT and Eyeball triage. Hazard ratios (HR) for DEPT and Eyeball triage were calculated using Cox regression.

Positive predictive value (PPV), negative predictive value (NPV), sensitivity and specificity were calculated with standard methods for DEPT and Eyeball triage using grouping into either green/yellow or orange/red patient groups. Kappa statistics were used to describe the agreement between DEPT and Eyeball triage. Kappa values were evaluated using the descriptions from Landis and Koch. ${ }^{14}$

Data were analysed using SPSS Statistics Version 22 (IBM, 2013).

\section{RESULTS}

During the study period 6383 encounters were recorded with a total of 5568 unique patients. DEPT was performed for 6290 $(98.5 \%)$ and Eyeball triage for $6382(\sim 100 \%)$ of the patient visits. A total of 6290 encounters received both triage assessments and were included in the statistical analysis. Of the included sample, $3116(49.5 \%)$ were male and the overall mean (SD) age was 60.2 (20.0) years. According to DEPT, 38 patients $(0.6 \%)$ were categorised as red, $1704(27.1 \%)$ as orange, $2487(39.5 \%)$ as yellow and 2061 patients $(32.8 \%)$ as 


\begin{tabular}{|c|c|}
\hline & Total sample \\
\hline Total, $\mathrm{n}$ & 6290 \\
\hline \multicolumn{2}{|l|}{ Demographics } \\
\hline Gender, male $(n, \%)$ & $3116(49.5)$ \\
\hline Age, years, mean (SD) & $60.2(20.0)$ \\
\hline \multicolumn{2}{|l|}{ Vital signs } \\
\hline \multicolumn{2}{|l|}{ Blood pressure $(\mathrm{mmHg}, 95 \% \mathrm{Cl})$} \\
\hline Systolic blood pressure & 134.1 (133.3 to 134.8$)$ \\
\hline Diastolic blood pressure & 75.8 (75.3 to 76.2 ) \\
\hline Heart rate $(/ \mathrm{min}, 95 \% \mathrm{Cl})$ & 81.5 (80.9 to 82.0$)$ \\
\hline Respiratory rate $(/ \mathrm{min}, 95 \% \mathrm{Cl})$ & 17.1 (17.0 to 17.2$)$ \\
\hline $\mathrm{SpO}_{2}(\%, 95 \% \mathrm{Cl})$ & 95.8 (95.5 to 96.1$)$ \\
\hline Temperature $\left({ }^{\circ} \mathrm{C}, 95 \% \mathrm{Cl}\right)$ & 36.2 (36.0 to 36.3 ) \\
\hline \multicolumn{2}{|l|}{ Comorbidity } \\
\hline Previous MI (n, \%) & $405(6.4)$ \\
\hline $\mathrm{PCl}(\mathrm{n}, \%)$ & $282(4.5)$ \\
\hline CABG $(n, \%)$ & $209(3.3)$ \\
\hline Heart failure $(n, \%)$ & $369(5.9)$ \\
\hline Atrial fibrillation (n, \%) & $694(11)$ \\
\hline Hypertension (n, \%) & $1404(22.3)$ \\
\hline Diabetes $(n, \%)$ & $672(10.7)$ \\
\hline $\operatorname{COPD}(n, \%)$ & $530(8.4)$ \\
\hline Liver disease $(n, \%)$ & $116(1.8)$ \\
\hline Renal disease $(n, \%)$ & $230(3.7)$ \\
\hline \multicolumn{2}{|l|}{ Cancer $(\mathrm{n}, \%)$} \\
\hline Former & $490(7.8)$ \\
\hline Current & $461(7.3)$ \\
\hline History of stroke $(n, \%)$ & $495(7.9)$ \\
\hline \multicolumn{2}{|l|}{ Smoking $(\mathrm{n}, \%)$} \\
\hline Previous & $1388(22.1)$ \\
\hline Active & $1541(24.5)$ \\
\hline Never & $1997(31.8)$ \\
\hline $\mathrm{n} / \mathrm{a}$ & $1364(21.7)$ \\
\hline
\end{tabular}

CABG, coronary artery bypass grafting; $C O P D$, chronic obstructive pulmonary disease; $\mathrm{MI}$, myocardial infarction; $\mathrm{PCl}$, percutaneous coronary intervention; $\mathrm{SpO}_{2^{\prime}}$ peripheral oxygen saturation.

green. According to Eyeball triage, 118 patients (1.9\%) were categorised as red, $1016(16.2 \%)$ as orange, $2804(44.6 \%)$ as yellow and 2352 patients $(37.4 \%)$ as green $(\mathrm{P}<0.001$ for difference between triage methods).

The baseline characteristics of the overall population are shown in table 1. Baseline characteristics for patients in the different DEPT and Eyeball triage categories are available in the online supplementary appendix table 1.1 and table 1.2).

\section{8-hour mortality}

Within 48 hours, $58(0.9 \%)$ of the patients died. Mortality in relation to triage category for both DEPT and Eyeball triage is shown in table 2 .

With only one exception (patients in the yellow DEPT group), the HR for death increased with higher level of triage category for both DEPT and Eyeball triage, with Eyeball triage being the best discriminator of the two (table 2).

\section{0-day mortality}

Of the total 6290 patient visits, 263 (4.2\%) died within 30 days. Mortality in relation to triage category as well as HR is shown in table 3.

Cox analyses of 30-day mortality showed that the HR for patients increased with triage category for both DEPT and Eyeball triage (table 3).

Test characteristics for DEPT and Eyeball triage in relation to prediction of patients at high and low risk of 30-day mortality is shown in table 4.

Eyeball triage had an overall higher sensitivity and specificity as well as a better positive and negative predictive value in terms of predicting patients at high and low risk of 30-day mortality. The same was found for 48-hour mortality. These data are available in the online supplementary appendix table 2 .

\section{Agreement between DEPT and Eyeball triage}

The agreement between DEPT and Eyeball triage was found to be 0.05 (95\% CI 0.04 to 0.07 ) using unweighted kappa statistics, which is regarded as very low agreement. ${ }^{14}$ Linear weighted kappa was found to be slightly higher but still represents a very low level of agreement of 0.10 (95\% CI 0.08 to 0.12$).{ }^{14}$

Table 5 shows the distribution of patients between DEPT and Eyeball triage.

\section{DISCUSSION}

Triage is an essential element of modern emergency care and traditionally the triage process has been an integral element of ED nursing practice. In this prospective study we found that a simple clinical assessment by non-clinician hospital staff was a significantly better predictor of death in an unselected ED patient group. Eyeball triage was a better discriminator for 48-hour and 30-day mortality for all triage groups than DEPT. We also found the agreement between DEPT and Eyeball triage to be very low.

There have been few reports on how a clinical assessment compares to formalised triage in predicting patient outcome. One study by Storm-Versloot et a ${ }^{15}$ compared the two triage algorithms Emergency Severity Index and Manchester Triage System with a clinical assessment by a triage nurse. The study investigated predictive performance for all three triage methods in relation to resource use and length of stay and found no

Table 2 48-Hour mortality and hazard ratios (HR) in relation to 48-hour mortality for Danish Emergency Process Triage (DEPT) and Eyeball triage category

\begin{tabular}{|c|c|c|c|c|c|}
\hline & Red & Orange & Yellow & Green & Total \\
\hline DEPT & 38 & 1704 & 2487 & 2061 & 6290 \\
\hline Deaths, n (\%) & $7(18.4 \%)$ & $24(1.4 \%)$ & $14(0.6 \%)$ & $13(0.6 \%)$ & $58(0.9 \%)$ \\
\hline $\mathrm{HR}(95 \% \mathrm{Cl})$ & 30.9 (12.3 to 77.4$)$ & 2.2 (1.1 to 4.4$)$ & 0.9 (0.4 to 1.9$)$ & 1 & \\
\hline Eyeball & 118 & 1016 & 2804 & 2352 & 6290 \\
\hline Deaths, n (\%) & $18(15.3 \%)$ & $22(2.2 \%)$ & $15(0.5 \%)$ & $3(0.1 \%)$ & $58(0.9 \%)$ \\
\hline $\mathrm{HR}(95 \% \mathrm{Cl})$ & 128.7 (37.9 to 436.8$)$ & 17.1 (5.1 to 57.1$)$ & 4.2 (1.2 to 14.6$)$ & 1 & \\
\hline
\end{tabular}

'Total' refers to the total number of patient visits in each triage category. 'Deaths' refers to the number of deaths within 48 hours. 
Table 3 30-Day mortality and hazard ratios (HR) in relation to 30-day mortality for Danish Emergency Process Triage (DEPT) category and Eyeball triage category

\begin{tabular}{|c|c|c|c|c|c|}
\hline & Red & Orange & Yellow & Green & Total \\
\hline DEPT & 38 & 1704 & 2487 & 2061 & 6290 \\
\hline Deaths, n (\%) & $13(34.2 \%)$ & $102(6.0 \%)$ & $99(4.0 \%)$ & $49(2.4 \%)$ & $263(4.2 \%)$ \\
\hline $\operatorname{HR}(95 \% \mathrm{Cl})$ & 19.1 (10.4 to 35.2$)$ & 2.6 (1.8 to 3.6$)$ & 1.7 (1.2 to 2.4$)$ & 1 & \\
\hline Eyeball triage & 118 & 1016 & 2804 & 2352 & 6290 \\
\hline Deaths, n (\%) & $36(30.5 \%)$ & $103(10.1 \%)$ & $91(3.3 \%)$ & $33(1.4 \%)$ & $263(4.2 \%)$ \\
\hline $\mathrm{HR}(95 \% \mathrm{Cl})$ & 27.1 (16.9 to 43.5$)$ & 7.6 (5.1 to 11.2 ) & 2.4 (1.6 to 3.5$)$ & 1 & \\
\hline
\end{tabular}

'Total' refers to the total number of patient visits in each triage category. 'Deaths' refers to the number of deaths within 30 days.

significant difference between them. They also investigated the effectiveness of triage using a reference standard derived from expert opinion and found that informally structured triage had the highest level of agreement. Despite this finding, the study concluded that it was preferable to use a formalised triage algorithm since it assured uniformity and transparency in the triage decisions. ${ }^{15}$ Although triage algorithms provide some level of transparency, we were not able to identify studies that investigated whether the reliability (ie, interobserver agreement) of formalised triage is greater than that of informally structured triage. Current studies investigating interobserver agreement of triage algorithms report heterogeneous results ranging from slight to almost perfect agreement. ${ }^{4}$

The existing literature on limitations in the validation of triage scales points out that examining validity of triage is generally quite difficult. As previously stated by Twomey et al, ${ }^{6}$ "validity refers to the degree with which the measured acuity level reflects the patient's true acuity at the time of triage. The term valid implies that there is some sort of external reference or 'gold standard' which by definition has absolute accuracy." No such measure exits for triage. Validation of DEPT is related to precursors of the model where triage levels have been shown to be associated with mortality and admission to the intensive care unit. $^{7}$ This kind of 'construct validity' is the most common in validation of triage scales. ${ }^{78}$ In this study we chose to investigate the relation between triage category and mortality in the belief that this measure best reflects acuity. Even though it is unknown if introducing triage can actually reduce patient mortality, a successful triage tool should be able to detect patients at high risk of short-term mortality.

To our knowledge, no previous studies have investigated the relationship between DEPT and mortality in the ED. ${ }^{7}$ However, one study by Barfod et $a l^{12} 16$ investigated the relationship between the first Danish modified ADAPT model and in-hospital mortality in a comparable unselected ED patient group.
The study had a similar distribution of patients to ADAPT categories as ours to DEPT categories, but with fewer patients in the orange triage group and more patients in the red triage group. ${ }^{9}$ The study found an overall 28-day mortality which was equal to the overall 30-day mortality found in our cohort. Barfod et al also investigated the Odds ratio for in-hospital mortality in relation to ADAPT; the results were similar and with overlapping confidence intervals to our calculated HR for 30-day mortality in relation to DEPT.

Several studies have addressed the issue of time-consuming triage algorithms, ${ }^{717}$ but only a few have investigated the associated time spent on performing formalised triage. ${ }^{18} 19$ This is intriguing since an inefficient triage system could lead to ED crowding which is known to be a negative prognostic factor in relation to patient outcome. ${ }^{20} \mathrm{~A}$ previous retrospective cross-sectional study conducted at an urban academic ED in the USA found that less than half of high-acuity patients completed triage within time frames recommended by the Emergency Severity Index, concluding that the value and safety of the triage process should be reassessed. ${ }^{21}$ As has previously been stated, "being quickly assessed in a treatment bed is better than sitting in a waiting room not receiving any care". ${ }^{22}$

Different approaches to optimising triage have been investigated. Previous research has documented that implementing additional triage officers in the triage units will improve efficiency and implementing physician-assisted triage could decrease ED length of stay. ${ }^{1723-25}$ It is yet to be investigated if simply applying Eyeball triage could result in similar results.

\section{Strengths and limitations of this study}

While previous validation studies of formalised triage have mainly focused on standardised patient scenarios or predictive validity for surrogate measures of acuity, we chose to sample 'real-life' patients and compared triage categories with an

Table 4 Prognostic performance of Danish Emergency Process Triage (DEPT) and Eyeball triage. Discriminatory ability for DEPT and Eyeball triage in relation to prediction of patients in high and low risk of 30-day mortality when using grouping into either green/yellow or orange/red patient groups

\begin{tabular}{|c|c|c|c|c|c|c|}
\hline & $\begin{array}{l}\text { 30-day mortality, } \\
n=263(100 \%)\end{array}$ & $\begin{array}{l}\text { Sensitivity \% } \\
(95 \% \mathrm{Cl})\end{array}$ & $\begin{array}{l}\text { Specificity \% } \\
(95 \% \mathrm{Cl})\end{array}$ & $\begin{array}{l}\text { NPV \% } \\
(95 \% \mathrm{Cl}) \\
\end{array}$ & $\begin{array}{l}\text { PPV \% } \\
(95 \% \mathrm{Cl})\end{array}$ & Accuracy (\%) \\
\hline \multicolumn{7}{|l|}{ DEPT } \\
\hline Orange/red & $115(43.7 \%)$ & 43.7 (37.9 to 49.8$)$ & 73.0 (71.9 to 74.1$)$ & 96.7 (96.2 to 97.2 ) & 6.6 (5.5 to 7.9 ) & 71.8 \\
\hline Green/yellow & $148(56.3 \%)$ & & & & & \\
\hline \multicolumn{7}{|l|}{ Eyeball triage } \\
\hline Orange/red & $139(52.9 \%)$ & 52.9 (46.8 to 58.8 ) & 83.5 (82.5 to 84.4$)$ & 97.6 (97.1 to 98.0$)$ & 12.3 (10.5 to 14.3$)$ & 82.2 \\
\hline Green/yellow & $124(47.1 \%)$ & & & & & \\
\hline
\end{tabular}

Percentages may not equal $100 \%$ due to rounding.

PPV, positive predictive value; NPV, negative predictive value.

$p<0.05$ for all. 
Table 5 Association between Danish Emergency Process Triage and Eyeball triage categories in emergency department patients

\begin{tabular}{|c|c|c|c|c|c|}
\hline DEPT Eyeball & Green & Yellow & Orange & Red & Total \\
\hline Green & 857 (13.6\%) & 925 & 256 & 23 & $32.8 \%$ \\
\hline Yellow & 994 & $1110(17.7 \%)$ & 359 & 24 & $39.5 \%$ \\
\hline Orange & 500 & 767 & $387(6.2 \%)$ & 50 & $27.1 \%$ \\
\hline Red & 1 & 2 & 14 & $21(0.3 \%)$ & $0.6 \%$ \\
\hline Total & $37.4 \%$ & $44.6 \%$ & $16.2 \%$ & $1.9 \%$ & $100 \%$ \\
\hline
\end{tabular}

objective endpoint. Since one of the key purposes of triage is to quantify risk and accelerate treatment for high-risk patient groups, 48-hour and 30-day mortality are important.

A further strength of the present study is the large unselected patient group and the high completion rate for both triage methods. Both triage methods were carried out in almost immediate succession, minimising the bias of initiated treatment, and Eyeball triage was performed by a phlebotomist with no formal training in patient evaluation. This was done in order to minimise selection bias due to prior knowledge of formalised triage scales or extensive experience with patients in the ED. We therefore believe that Eyeball triage could be implemented on a broader scale with at least the same accuracy in the prediction of mortality and possibly as a general measure for patient acuity.

This study has several limitations. First, treatment was initiated according to DEPT, and Eyeball triage had no consequences on the handling of the patient. This could possibly influence the phlebotomist into making more blunt triage decisions and not taking into account that a formalised triage system will generally choose a high sensitivity at the cost of a low specificity. ${ }^{16}$ The fact that the treatment was initiated using DEPT could also account for some of the explanation as to why mortality is lower in the orange and red DEPT groups compared with Eyeball triage. However, Eyeball triage showed a better discriminative ability through all patient groups for both 48-hour and 30-day mortality. Furthermore, we did not track the pattern of the individual triage officers and have not been able to identify differences related to each triage officer's level of experience or education. This could be a possible approach in future studies. As a last point, we want to emphasise that even though we believe mortality to be one of the best markers of acuity, some patients might present with acute symptoms but not be severely ill. This issue is related to most endpoints used in the common validation of triage scales.

\section{Clinical implications and future studies}

We believe the findings from our study indicate that we need new initiatives in order to evaluate triage and optimise initial patient stratification. It would be preferable to initiate randomised controlled trials investigating different triage methods with clinically relevant endpoints such as, for example, short-term mortality risk. Furthermore, the results from this study suggest that a clinical evaluation should potentially have a larger role in future triage algorithms.

\section{CONCLUSION}

Agreement between formalised triage and a quick clinical assessment in the form of Eyeball triage is poor. A simple clinical assessment by phlebotomists is superior to a formalised triage system to predict short-term mortality in ED patients.

\section{Author affiliations}

'Department of Obstetrics and Gynecology, Rigshospitalet, Kobenhavn, Denmark ${ }^{2}$ Department of Internal Medicine, Amager Hospital, Copenhagen, Denmark ${ }^{3}$ Department of Anesthesia, Centre of Head and Orthopedics Surgery, Rigshospitalet, University of Copenhagen, Copenhagen, Denmark

${ }^{4}$ Department of Cardiology, Rigshospitalet, University of Copenhagen, Copenhagen, Denmark

${ }^{5}$ Department of Clinical Biochemistry, North Zealand Hospital, University of Copenhagen, Hillerød, Denmark

${ }^{6}$ Department of Emergency Medicine and Prehospital Care, Helsingborg Hospital, Helsingborg, Sweden

${ }^{7}$ Clinical Research Centre, University of Copenhagen, Hvidovre, Denmark

${ }^{8}$ Department of Cardiology, Herlev Hospital, University of Copenhagen, Copenhagen, Denmark

Contributors KKI is the primary initiator of the 'Triage study' and wrote the first draft of the study protocol. AKSI wrote the manuscript, performed the statistical analyses and participated in the data collection. MK and RM $\varnothing$ participated in the data collection and critically revised the manuscript. LK, GS, JLF, JE-O, LSR and MS participated with interpretation of the data and critically reviewed the manuscript.

Funding The authors have not declared a specific grant for this research from any funding agency in the public, commercial or not-for-profit sectors

Competing interests None declared.

Patient consent Not required.

Ethics approval The study was conducted according to Danish ethical regulations and was approved by the Danish Data Protection Agency, journal number: HIH2013028.

Provenance and peer review Not commissioned; externally peer reviewed.

(c) Article author(s) (or their employer(s) unless otherwise stated in the text of the article) 2019. All rights reserved. No commercial use is permitted unless otherwise expressly granted.

\section{REFERENCES}

1 Aacharya RP, Gastmans C, Denier Y. Emergency department triage: an ethical analysis. BMC Emerg Med 2011;11:16.

2 Brabrand M, Folkestad L, Hallas P. Visitation og triage af akut indlagte medicinske patienter. Dan Med Bull 2010:1666-8.

3 Skriver C, Lauritzen MM, Forberg JL, et al. [Triage quickens the treatment of the most sick patients]. Ugeskr Laeger 2011;173:2490-3.

4 Farrohknia N, Castrén M, Ehrenberg A, et al. Emergency department triage scales and their components: a systematic review of the scientific evidence. Scand J Trauma Resusc Emerg Med 2011;19:42.

5 Moll HA. Challenges in the validation of triage systems at emergency departments. J Clin Epidemiol 2010;63:384-8.

6 Twomey M, Wallis LA, Myers JE. Limitations in validating emergency department triage scales. Emerg Med J 2007;24:477-9.

7 Iversen AK, Kristensen M, Østervig R, et al. [No evidence that formalized triage is superior to informally structured triage]. Ugeskr Laeger 2015;177.

8 Kuriyama A, Urushidani S, Nakayama T. Five-level emergency triage systems: variation in assessment of validity. Emerg Med J 2017;34:703-10.

9 Plesner LL, Iversen AK, Langkjær $S$, et al. The formation and design of the TRIAGE study-baseline data on 6005 consecutive patients admitted to hospital from the emergency department. Scand J Trauma Resusc Emerg Med 2015;23:106.

10 North Zealand Hospital. 2014 https://www.nordsjaellandshospital.dk

11 DEPT. User Manuel Danish Emergency Process Triage - DEPT. http://deptriage.dk/ brugervejledning/

12 Barfod C, Lauritzen MM, Danker JK, et al. The formation and design of the 'Acute Admission Database': a database including a prospective, observational cohort of 6279 patients triaged in the emergency department in a larger Danish hospital. Scand J Trauma Resusc Emerg Med 2012;20:29.

13 Nordberg M, Lethvall S, Castrén M. The validity of the triage system ADAPT. Scand J Trauma Resusc Emerg Med 2010;18:P36.

14 Landis JR, Koch GG. The measurement of observer agreement for categorical data. Biometrics 1977:33:159-74

15 Storm-Versloot MN, Ubbink DT, Kappelhof J, et al. Comparison of an informally structured triage system, the emergency severity index, and the Manchester triage system to distinguish patient priority in the emergency department. Acad Emerg Med 2011;18:822-9.

16 Barfod C, Lauritzen MM, Danker JK, et al. Abnormal vital signs are strong predictors for intensive care unit admission and in-hospital mortality in adults triaged in the emergency department: a prospective cohort study. Scand J Trauma Resusc Emerg Med 2012:20:28

17 Subash F, Dunn F, McNicholl B, et al. Team triage improves emergency department efficiency. Emerg Med J 2004;21:542-4 
18 Jiménez JG, Murray MJ, Beveridge R, et al. Implementation of the Canadian Emergency Department Triage and Acuity Scale (CTAS) in the Principality of Andorra: can triage parameters serve as emergency department quality indicators? CJEM 2003:5:315-22.

19 Storm-Versloot MN, Vermeulen H, van Lammeren N, et al. Influence of the Manchester triage system on waiting time, treatment time, length of stay and patient satisfaction: a before and after study. Emerg Med J 2014;31:13-18.

20 Bernstein SL, Aronsky D, Duseja R, et al. The effect of emergency department crowding on clinically oriented outcomes. Acad Emerg Med 2009;16:1-10.

21 Weber EJ, McAlpine I, Grimes B, et al. Mandatory triage does not identify high-acuity patients within recommended time frames. Ann Emerg Med 2011:58:137-42.
22 American College of Emergency Physicians. Approaching full capacity in the

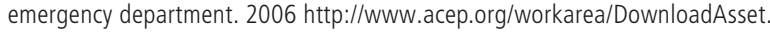
aspx?id=8852.

23 Partovi SN, Nelson BK, Bryan ED, et al. Faculty triage shortens emergency department length of stay. Acad Emerg Med 2001;8:990-5.

24 Choi YF, Wong TW, Lau CC. Triage rapid initial assessment by doctor (TRIAD) improves waiting time and processing time of the emergency department. Emerg Med J 2006:23:262-5.

25 Burström L, Nordberg M, Ornung G, et al. Physician-led team triage based on lean principles may be superior for efficiency and quality? A comparison of three emergency departments with different triage models. Scand I Trauma Resusc Emerg Med 2012;20:57. 